\title{
REPRESENTATION OF JORDAN AND LIE ALGEBRAS
}

\author{
BY \\ GARRETT BIRKHOFF AND PHILIP M. WHITMAN(')
}

1. Introduction. In a linear associative algebra $A$ one can introduce a new product $\theta(x, y)$ in terms of the given operations by setting

$$
\theta(x, y)=\alpha x y+\beta y x,
$$

where $\alpha$ and $\beta$ are scalars independent of $x$ and $y, \alpha$ and $\beta$ not both zero. Albert [3] observes ( ${ }^{2}$ ) that if a linear subset $S$ of $A$ is closed under $\theta$ (in particular, if $S$ is $A$ itself) then $S$ is of one of three types: $S$ is an associative algebra, closed under the given multiplication $x y$; or $S$ is a Lie algebra with product obtained by setting $\alpha=1$ and $\beta=-1$ in (1):

$$
[x, y]=y x-x y \text {; }
$$

or $S$ is a Jordan algebra with product obtained by setting $\alpha=\beta=1 / 2$ in (1):

$$
x \cdot y=(x y+y x) / 2 \text {. }
$$

We propose to study various generalizations, properties, and representations of Lie and Jordan algebras.

2. Definitions and problems. We begin with a generalization of the idea of equation (1) of the previous section.

Let $\mathfrak{A}$ be any set of abstract algebras $\left({ }^{3}\right)$ closed under given $n_{i}$-ary basic operations $f_{i}$, and let $\Theta$ be any set of operations $\theta_{1}, \cdots, \theta_{n}$ obtained by compounding the $f_{i}$. If $A \in \mathfrak{A}$, then the elements of $A$ together with the operations $\theta_{1}, \cdots, \theta_{n}$ form an algebra $\Theta(A)$. All algebras which can be obtained in this manner for fixed $\Theta$ [and hence fixed $\mathfrak{A}$ ] are called $\theta$-algebras. Clearly the definition of $\Theta(A)$ is also applicable to the case where $A$ is closed under the $\theta_{i}$ but not under the $f_{i}$; we consider examples of this in Theorems 8-10

Presented to the Society, August 23, 1946, under the title Representation theory for certain non-associative algebras; received by the editors December 8, 1947.

(1) This paper was presented in part to the Algebra Conference, University of Chicago, July, 1946. The junior author wishes to express his appreciation of a Frederick Gardner Cottrell Grant by the Research Corporation which assisted this research.

(2) In describing earlier work on the subject, it must be remembered that $A$ is usually given as an algebra of matrices or of linear transformations. We shall assume familiarity with the correspondence between associative algebras and algebras of matrices. Any algebra of matrices is associative under ordinary matrix multiplication, while (essentially through its "regular representation") every linear associative algebra is isomorphic to an algebra of matrices. The general theory is due to E. Noether [21]. Numbers in brackets refer to the references cited at the end of the paper.

( $\left.{ }^{3}\right)$ For the general theory of algebras, see G. Birkhoff [5], [8]; McKinsey and Tarski [18], [19], [22]. 
below. However, for the present we require closure under the $f_{i}$ as well.

By a Lie algebra we mean an algebra $L$ such that $L=\Theta(A)$ for some associative algebra $A$ when $\Theta=\left\{\theta_{1}, \theta_{2}\right\}$ where

$$
\theta_{1}(x, y)=x+y, \quad \theta_{2}(x, y)=y x-x y ;
$$

cf. (2).

By a special( $\left.{ }^{4}\right)$ Jordan algebra we mean an algebra $J$ such that $J=\Theta(A)$ for some associative algebra $A$ when $\Theta=\left\{\theta_{1}, \theta_{2}\right\}$ where

$$
\theta_{1}(x, y)=x+y, \quad \theta_{2}(x, y)=(x y+y x) / 2 ;
$$

cf. (3). The field of scalars of course must not be of characteristic 2.

In these two cases respectively, $\Theta(A)$ may be denoted $L(A)$ and $J(A)$.

Conversely, if $X$ is a given algebra (not necessarily associative) closed under its operations which are called $\theta_{1}, \theta_{2}, \cdots, \theta_{n}$ then we may seek to find an algebra $A$ consisting of the same elements as $X$ but with operations $f_{i}$, such that $X=\Theta(A)$. We may then say that we have exhibited $X$ as a $\theta$-algebra. In general, given an arbitrary $X$ and $\Theta$, it is not possible( $\left.{ }^{5}\right)$ to find an $A$ by means of which we may exhibit $X$ in the above sense. Hence, as usual in representation theory, we find it convenient to consider homomorphisms as well as isomorphisms, and to extend the meaning of our terms accordingly.

Definition. Let $X$ be a particular algebra with elements $x_{i}$ and operations $\theta_{1}, \cdots, \theta_{n}$, let $\mathfrak{A}$ be a set of algebras, and let $\bar{\theta}_{1}, \cdots, \bar{\theta}_{n}$ be specified as compound operations on the elements of the algebras of $\mathfrak{A}$. Then a $\theta$-embedding, or $\theta$-representation, of $X$ is a correspondence, $\alpha: x \rightarrow \alpha(x)$, from $X$ into $A \in \mathfrak{A}$, such that

$$
\alpha\left(\theta_{i}\left(x_{1}, \cdots, x_{m}\right)\right)=\bar{\theta}_{i}\left(\alpha\left(x_{1}\right), \cdots, \alpha\left(x_{m}\right)\right)
$$

for all $i$ and all $x_{1}, \cdots, x_{m} \in X$. We shall restrict ourselves to the case where $A$ is generated by the $\alpha\left(x_{i}\right)$.

The algebra $A$ is then called a $\theta$-envelope of $X$.

The $\theta$-embedding is called an iso- $\theta$-embedding if the correspondence $x \rightarrow \alpha(x)$ is one-to-one, and then $A$ is an iso- $\theta$-envelope of $X$. then

If $\alpha_{1}, \alpha_{2}, \cdots$ are $\theta$-embeddings of $X$, in $\theta$-envelopes $\left\{A_{1}, A_{2}, \cdots\right\} \in \mathfrak{X}$

$$
x \rightarrow \alpha(x) \equiv\left(\alpha_{1}(x), \alpha_{2}(x), \cdots\right)
$$

is a $\theta$-embedding of $X$ in a subalgebra $A_{u}(X)$ of the direct union $A_{1} \otimes A_{2} \otimes \cdots$, namely, the subalgebra generated by the set of all $\left(\alpha_{1}\left(x_{i}\right), \alpha_{2}\left(x_{i}\right), \cdots\right)$. But $\alpha_{n}$

(4) The word special (sometimes concrete) is used to distinguish these algebras from those defined by identities without reference to an associative algebra; cf. Albert [4], Kalisch [16]. The question remains open whether special Jordan algebras can be defined by identities.

(b) For example, if $\Theta$ is the set of operations (5), it is known that the identity $a^{2}(b a)$ $=\left(a^{2} b\right) a$ must be satisfied in $X$; cf. [15]. This does not, however, exclude the possibility of a comparable representation if other operations than the given $f_{i}$ are taken as basic. 
is a homomorphic image of $\alpha$, in the sense that if $\alpha\left(x_{i}\right)=\alpha\left(x_{j}\right)$, then $\alpha_{n}\left(x_{i}\right)$ $=\alpha_{n}\left(x_{j}\right)$, while by (6) and (7), both $\alpha$ and $\alpha_{n}$ are homomorphisms with respect to the $\theta_{i}$. Hence we have the following theorem.

THEOREM 1. If I is closed under the operations of taking direct unions and subalgebras then there exists $A_{u}(X) \in \mathfrak{X}$ such that there is a $\theta$-embedding $\theta_{u}$ of $X$ in $A_{u}(X)$ and any other $\theta$-embedding of $X$ in an algebra of $\mathfrak{X}$ is a homomorphic image of $\theta_{u}$.

Definition. $\theta_{u}$ is called the universal $\theta$-embedding of $X$ in $\mathfrak{A}$, and $A_{u}(X)$ is called the free $\theta$-envelope of $X$ [with reference to $\mathfrak{A}$ ] or universal $\theta$-algebra of $X$. In the particular cases of equations (4) and (5) we shall speak respectively of Lie embeddings and envelopes and Jordan embeddings and envelopes.

Evidently $A_{u}(X)$ is the free algebra of $\mathfrak{A}$ in the sense that every $A_{\mathfrak{i}} \in \mathfrak{A}$ is a homomorphic image of $A_{u}(X)$, since the correspondence

$$
\left[\left(a_{1}, a_{2}, \cdots, a_{i-1}, a_{i}, a_{i+1}, \cdots\right) \in A_{u}(X)\right] \rightarrow\left[a_{i} \in A_{i}\right]
$$

is obviously such a homomorphism.

Then by applying the same homomorphism to the compound expressions in the operations of $\mathfrak{U}$ which define the $\theta_{i}$, we see that $\Theta\left(A_{i}\right)$ is a homomorphic image of $\Theta\left(A_{u}\right)$. Hence $\Theta\left(A_{u}\right)$ is a free algebra for $\Theta(A), A \in \mathfrak{A}$.

If some $\theta$-embedding of $X$ is one-to-one, $X$ is called a $\theta$-algebra. In this case we may identify the elements of $X$ with their images in $A$.

If $X$ is a $\theta$-algebra, then $\theta_{u}$ must be one-to-one, for if it were many-to-one, so would be each other embedding (the latter being a homomorphic image of $\theta_{u}$ ) contrary to hypothesis.

Among the problems which may be stated are these: When is $X$ a $\theta$-algebra? What identities are satisfied by $\theta$-algebras for a given $\Theta$, in particular by special Jordan algebras? Is the set of all $\theta$-algebras, for given $\Theta$, closed under the operations of taking subalgebras, direct unions, and homomorphic images?

As far as concerns subalgebras, it is obvious that the answer is yes: if $Y$ is a subalgebra of $X$, and $X=\Theta(A)$, then we need only take the subalgebra $B$ of $A$ generated by the images of elements of $Y$, and $Y=\Theta(B)$.

Likewise, suppose $X_{1}, X_{2}, \cdots$ are given to be $\theta$-algebras: $X_{i}=\Theta\left(A_{i}\right)$, $A_{i} \in \mathfrak{A}$. Then $X_{1} \otimes X_{2} \otimes \cdots$ can be put into one-to-one correspondence with the subset of $A_{1} \otimes A_{2} \otimes \cdots$ consisting of the $\left(\alpha_{1}\left(x_{i}\right), \alpha_{2}\left(x_{j}\right), \cdots\right)$, and the latter generates $A_{1} \otimes A_{2} \otimes \cdots$. Then

$$
\Theta\left(A_{1} \otimes A_{2} \otimes \cdots\right)=\Theta\left(A_{1}\right) \otimes \Theta\left(A_{2}\right) \otimes \cdots=X_{1} \otimes X_{2} \otimes \cdots .
$$

Thus this set of $\theta$-algebras is closed under subalgebras and direct unions. Closure under the taking of homomorphic images remains undecided. 
It is known [8, p. 324] that the set $\mathfrak{A}$ is definable by identities if and only if it is closed under the operations of taking subalgebras, direct unions, and homomorphic images.

Consider the congruence relation $\phi_{i}$ on $A_{u}(X)$ which identifies elements $\left(b_{1}, b_{2}, \cdots\right)$ and $\left(c_{1}, c_{2}, \cdots\right)$ of $A_{u}(X)$ if and only if $b_{i}=c_{i}$ for fixed $i$. As before, the algebra obtained by this identification is precisely $A_{i}$. In the case of linear algebras (including Lie, Jordan, and associative algebras) any congruence relation $\phi_{i}$ on $A_{u}(X)$ is determined by the set $K_{i}$ of elements which are congruent to 0 . Clearly $K_{i}$ is a (two-sided) ideal; hence we may write $A_{i}=A_{u}(X) / K_{i}$.

It is natural to ask: for what $K$ is $A_{u}(X) / K$ an iso- $\theta$-envelope of $X$ ? Also we may seek to determine $A_{u}(X)$ for various $X$; in particular we would like to know how to solve the decision problem for $X$.

3. A general property related to $\theta$-embeddings. We consider a canonical form for polynomials in a linear (not necessarily associative) algebra.

Theorem 2. In a linear algebra, any polynomial in elements $e_{i}$ can be expressed as a linear combination of monomials $e_{i(1)}^{p(1)} e_{i(2)}^{p(2)} \cdots e_{i(r)}^{p(r)}$ with $i(1)<i(2)$ $<\cdots<i(r)$, provided

$$
e_{j} e_{i}=\lambda_{i j} e_{i} e_{j}+\sum_{k} c_{k}^{i j} e_{k}
$$

where the $\lambda_{i j}$ and $c_{\boldsymbol{x}}^{i y}$ are scalars.

Proof. Just as in the corresponding proof in [6], we can systematically shift the $e_{i}$ with small subscripts to the left by introducing terms of lower degree and using induction. It is not guaranteed that the result of this procedure is unique.

This shows that the usual theory for noncommutative polynomials in one variable (Jacobson [14]) can be applied also to noncommutative polynomials in several variables. The scalars $\lambda_{i j}$ and $c_{k}^{i j}$ do not have to be permutable with the $e_{i}$; we can have $e_{i} \lambda=\phi_{i}(\lambda) e_{i}$.

4. Determination of Lie envelopes. We seek solutions, for Lie algebras, of some of the problems already mentioned.

Let $X$ be any linear algebra satisfying

$$
[x, y]+[y, x]=0 .
$$

Then $X$ has a basis (finite or infinite) of elements $e_{1}, e_{2}, \cdots$. Hence, for given $x$ and $y,[x, y]$ is some finite linear combination of the $e_{i}$. In particular, this applies if $x$ and $y$ are basis elements; say

$$
\left[e_{i}, e_{j}\right]=\sum_{k} c_{k}^{i j} e_{k}
$$

where only a finite number of $e_{n}$ enter into the sum (though the basis may be 
infinite), and the $c_{k}^{i j}$ are scalars ("structure constants"). From (2), we have in any Lie-envelope of $X$,

where $e_{k}^{\prime}$ is the image of $e_{k}: e_{k}^{\prime}=\alpha\left(e_{k}\right)$.

$$
e_{j}^{\prime} e_{i}^{\prime}=e_{i}^{\prime} e_{j}^{\prime}+\sum_{k} c_{k}^{i j} e_{k}^{\prime}
$$

Then Theorem 2 can be applied. It was shown in [6] and [24] that if

$$
[[x, y], z]+[[y, z], x]+[[z, x], y]=0
$$

also holds (so that $X$ is a Lie algebra) then this construction produces a linear associative algebra $L_{u}(X)$ with infinite basis, and that $X$ is the Lie algebra obtained from $L_{u}(X)$ by (2). Moreover, it is apparent from the construction that elements have been identified in $L_{u}(X)$ only when required by the hypothesis; hence, any other Lie-embedding of $X$ is a homomorphic image of $L_{u}(X)$. Hence, in the previous notation, $L_{u}(X)$ is $A_{u}(X)$.

Thus every linear algebra satisfying (8) and (9) has an iso-Lie-embedding in a linear associative algebra. In fact [6, Theorem 2] all Lie-embeddings have been determined by rational methods. Conversely, starting with a linear associative algebra and using $[x, y]=y x-x y$ we get an algebra in which these laws hold. Thus the embedding problem for Lie algebras has been solved.

However, we do not obtain in the abstract case the theorem of I. Ado [1] and E. Cartan [10] that every linear algebra with finite basis over the real or complex field satisfying (8)-(9) has an isomorphic Lie-embedding with a finite basis and conversely.

Let us consider an example of Lie-embedding.

THEOREM 3. Let $G$ be the regular representation of a Lie group, and let $X^{1}, \cdots, X^{r}$ be the differential operators defining its Lie algebra $L$. Then the free Lie-envelope of $L$ is isomorphic to the (associative) operator algebra generated by the $X^{i}$.

Proof. Define $G$ locally by canonical parameters; then the $X^{i}$ are analytic and (see for instance Campbell $[9$, p. 332])

$$
X^{i}=\partial / \partial x_{i}+\sum_{k} X_{k}^{i} \partial / \partial x_{k}, \quad X_{k}^{i}(0)=0
$$

Hence for any monomial in the $X^{\boldsymbol{i}}$,

$$
\left(X^{1}\right)^{n(1)} \cdots\left(X^{r}\right)^{n(r)}=\partial^{n(1)+\cdots+n(r)} / \partial x_{1}^{n(1)} \cdots \partial x_{r}^{n(r)}+\cdots,
$$

where the last dots represent terms in which the differential operators are either of order less than $n(1)+\cdots+n(r)$ or else have coefficients which vanish at the group identity 0 . Thus any monomial in the $X^{i}$ is a linear combination of monomials of equal or lower degree, and the associative algebra is isomorphic to the free Lie-envelope. 
We consider next a generalization of Hilbert's Basis Theorem.

TheOREM 4. Let $R$ be the ring of formal polynomials in $e_{1}, \cdots, e_{n}$ (n finite), with scalars in the field $F$, subject to the law

$$
e_{j} e_{i}=e_{i} e_{i}+\sum_{k} c_{k}^{i j} e_{k}
$$

Let $J$ be a left ideal of $R$. Then $J$ has a finite ideal basis.

Proof. Let $H_{n}(J)$ be the set of those homogeneous polynomial forms $q$ of degree $n$ such that $q+r \in J$ for some $r$ of degree less than $n$ (that is, let $H_{n}(J)$ be the set of "leading constituents" of degree $n$ of the polynomials in $J)$. We observe that each $H_{n}$, with 0 adjoined, is a linear subspace, since the sum or difference of two homogeneous polynomials of degree $n$ either is again such a polynomial or is 0 , and likewise for the product of such a polynomial by a scalar, while if $q_{1}+r_{1}$ and $q_{2}+r_{2}$ are in $J$, so are $\left(q_{1} \pm q_{2}\right)+\left(r_{1} \pm r_{2}\right)$ and $c\left(q_{i}+r_{i}\right)=c q_{i}+c r_{i}$, since $J$ is an ideal.

Let $H(J)$ be the set of polynomial forms comprising the linear subspace generated by the $H_{n}(J)(n=0,1,2, \cdots)$. We assert that the elements of $H(J)$, taken as polynomial forms in either the ring $R$ or the ring $K$ of commutative polynomials generated by $e_{1}, \cdots, e_{n}$, are an ideal. For if $h_{1}$, $h_{2} \in H(J)$, so do $c h_{1}$ and $h_{1}+h_{2}$ since $H(J)$ is a linear space, while if $h \in H(J)$ and $q \in R$ or $K$, then write $q$ and $h$ as sums of homogeneous polynomials

$$
q=q_{1}+\cdots+q_{s}, h=h_{1}+\cdots+h_{t}, h_{i} \in H_{f(i)}(J), d\left(q_{i}\right)=d_{i}
$$

where $d(p)$ is the degree of $p$. Then $q h=\sum_{i, j} q_{i} h_{j}$. But $q_{i} h_{j}$ is homogeneous of degree $d_{i}+f(j)$. Also $h_{j} \in H_{f(j)}$ implies $h_{j}+r \in J$ for some $r$ of degree less than $f(j)$. Then $q_{i} r$ is of degree less than $d_{i}+f(j)$, and $q_{i} h_{j}+q_{i} r=q_{i}\left(h_{j}+r\right) \in J$ since $J$ is an ideal. Thus by construction $g_{i} h_{j} \in H_{d_{i}+f(j)}(J)$. Hence, $q h \in H(J)$ since $H(J)$ is a linear space. Thus $H(J)$ is an ideal. In this regard the only difference between $H(J)$ as a subspace of $R$ and as a subspace of $K$ is that different polynomials are identified in the two cases.

Let $H^{*}$ denote the elements of the ideal $H(J)$, taken as polynomials in the ring $K$ of commutative polynomials in $e_{1}, \cdots, e_{n}$. By Hilbert's Basis Theorem $\left(^{6}\right), H^{*}$ has a finite ideal-basis $p_{1}^{*}, \cdots, p_{m}^{*}$. Since $H(J)$ and $H^{*}$ contain the same polynomial forms, we may consider $p_{1}^{*}, \cdots, p_{m}^{*}$ as belonging also to $H(J)$, in which case we shall denote them $p_{1}, \cdots, p_{m}$.

Thus $p_{i} \in H(J)$; by construction of $H, p_{i}$ is a linear combination of elements of various $H_{j}(J)$ :

$$
p_{i}=\sum_{j} b_{j}^{i} q_{j} ; \quad q_{j} \in H_{j}(J) ; \quad b_{j}^{i} \in F .
$$

(6) See, for instance, van der Waerden [23]. 
But by construction of $H_{j}$, given $q_{j} \in H_{j}(J)$ then there exists $r_{j}$ of degree less than $j$ such that $q_{j}+r_{j} \in J$. Set

$$
t_{i} \equiv \sum_{j} b_{j}^{i}\left(q_{j}+r_{j}\right)
$$

Then $t_{i} \in J$. Let $U$ be the ideal of $R$ generated by the $t_{i}$. Then $U \subset J$.

Hence, $H_{n}(U) \subset H_{n}(J)$ for all $n$. But suppose $h \in H_{n}(J)$. Then $h^{*} \in H^{*}$, where $h^{*}$ is the same polynomial form as $h$. Hence, $h^{*}=\sum_{j} y_{j}^{*} p_{j}^{*}, y_{j}^{*} \in H^{*}$. Let $y_{j}$ be the polynomial form in $H$ which becomes $y_{j}^{*}$ in $H^{*}$. Then by (10), $\sum_{j} y_{j} p_{j}$ has the same leading constituent as $h^{*}$, and hence as $h$.

But by construction the $t_{i}$ have the same leading constituents as the $p_{i}$, hence $\sum_{j} y_{j} t_{i}$ has the same leading constituent as the given $h$. Thus $H_{n}(J)$ $C H_{n}(U)$ so $H_{n}(J)=H_{n}(U)$ for all $n$.

The proof is then completed by the following lemma.

Lemma. Let $S$ be a subspace of a subspace $J$ of $R$. If $H_{n}(S)=H_{n}(J)$ for all $n$, then $S=J$.

Proof. Suppose $S<J$. Then there would exist a polynomial $p$ of lowest degree $n$ ( $n>0$ since $S$ includes 0$)$ which is in $J$ but not in $S$.

But $H_{n}(S)=H_{n}(J)$ contains all leading constituents of elements of $J$. Let $q$ be the leading constituent of $p$. Then $q \in H_{n}(J)$. But $H_{n}(S)=H_{n}(J)$, so $q \in H_{n}(S)$ also. Hence there exists $r$, of lower degree than $q$, such that $q+r \in S$. Then $p-(q+r)$ is of lower degree than $p$, since $p$ and $q+r$ have the same leading constituent. Hence $p-(q+r) \in S$ by hypothesis on $p$. But $q+r \in S$, $p=p-(q+r)+(q+r) \in S$, contrary to assumption that $p \notin S$. Thus the proof is completed.

5. Determination of Jordans envelopes. Having discussed the free Lieenvelopes of various algebras, we turn now to free Jordan envelopes. In this case let us denote $A_{u}(X)$ by $J_{u}(X)$. In all work with Jordan algebras, we require the base field to be of characteristic not 2 , in view of (3).

THEOREM 5. If $X$ has a finite basis, so does $J_{u}(X)$.

Proof. Let $X$ have the finite basis $e_{1}, \cdots, e_{n}$. Then $J_{u}(X)$ is generated by the monomials in $e_{1}, \cdots, e_{n}$ by Theorem 2 . Moreover,

$$
\sum_{k} c_{k}^{i i} e_{k}=e_{i} \cdot e_{i}=\left(e_{i} e_{i}+e_{i} e_{i}\right) / 2=e_{i} e_{i}
$$

so square-free monomials generate $J_{u}(X)$. Since $n$ is finite, $J_{u}(X)$ has a basis of not more than $2^{n}-1$ elements.

No general solution of the decision problem for $J_{u}(X)$ is known, but in special cases a solution can be found. It is readily verified that a zero, idempotent, or unit in $A$ has the same property in $J_{u}(X)$ and conversely. We con- 
sider several examples of Jordan envelopes, mostly of "irreducible $r$-number algebras" $[15$, p. 63$]$.

As one example, let $X$ be the free zero-algebra with basis $e_{1}, \cdots, e_{n}$. By (3), $e_{i} e_{j}+e_{j} e_{i}=2 e_{i} \cdot e_{j}=0$ for all $i, j$. In particular, let $i=j$. Then $e_{i}^{2}=0$. Hence, the free Jordan envelope of the free zero-algebra is an ideal of order $2^{n}-1$, excluding the unit element, in the extensive algebra of Grassmann $\left({ }^{7}\right)$.

Again, let $X$ be the algebra with basis $e_{0}, e_{1}, \cdots, e_{n}$, with

$$
\begin{aligned}
e_{0} \cdot e_{i} & =e_{i} \cdot e_{0}=e_{i} & (i=0, \cdots, n), \\
e_{i} \cdot e_{j} & =0 & (i \neq j ; i, j=1, \cdots, n), \\
e_{i} \cdot e_{i} & =-e_{0} & (i \neq 0) .
\end{aligned}
$$

Then the free Jordan envelope is the Clifford numbers $\left({ }^{8}\right)$.

THEOREM 6. If $X$ is the (special) Jordan algebra of all $n$ by $n$ matrices $(n \geqq 2)$ then $J_{u}(X)$ is the direct sum $M \oplus M$, where $M$ is the ordinary associative algebra of all $n$ by $n$ matrices.

Proof. Let $e_{i j}$ be the matrix with a single 1 , in the $i$ th row and $j$ th column, and zeros elsewhere. In $X$, the multiplication table is, by (3),

$$
e_{i j} \cdot e_{k l}=\left(\delta_{j k} e_{i l}+\delta_{i l} e_{k j}\right) / 2 \text {. }
$$

Set

$$
a_{i j} \equiv e_{i j} e_{j j}(i \neq j) ; \quad a_{i i} \equiv a_{i j} e_{j i} \equiv e_{i j} e_{j j} e_{j i}(i \neq j) ; \quad b_{i j} \equiv e_{j i}-a_{j i} ;
$$

$a_{i i}$ will be shown in (17) to be independent of $j$. We seek to obtain the multiplication table for $J_{u}(X)$.

$$
e_{i j} e_{k k}=0 \quad(k \neq i, j),
$$

for, since $e_{k k}$ is idempotent,

$$
\begin{aligned}
e_{i j} e_{k k} & =e_{i j} e_{k k} e_{k k}=\left\{2\left(e_{i j} \cdot e_{k k}\right)-e_{k k} e_{i j}\right\} e_{k k}=\left(0-e_{k k} e_{i j}\right) e_{k k} \\
& =-e_{k k}\left(e_{i j} e_{k k}\right)=-e_{k k}\left(0-e_{k k} e_{i j}\right)=e_{k k} e_{k k} e_{i j}=e_{k k} e_{i j} ;
\end{aligned}
$$

but also $e_{i j} e_{k k}=0-e_{k k} e_{i j}$; adding, $e_{i j} e_{k k}=0$. Likewise,

$$
\begin{aligned}
& e_{k k} e_{i j}=0 \\
& e_{i j} e_{i j}=0
\end{aligned}
$$

$$
\begin{array}{r}
(k \neq i, j) . \\
(i \neq j),
\end{array}
$$

for $e_{i j} e_{i j}=0-e_{i j} e_{i j}$ by (11).

$$
e_{i j} e_{j k}=e_{i i} e_{i k} \quad(i, j, k \text { all distinct }),
$$

for by (14),

(7) See, for instance, Chevalley [11, p. 145].

(8) See, for instance, Chevalley [11, p. 61]. 


$$
\begin{aligned}
0 & =e_{i j}\left(e_{i i} e_{j k}\right)=\left(e_{i j} e_{i i}\right) e_{j k}=\left(e_{i j}-e_{i i} e_{i j}\right) e_{j k} \\
& =e_{i j} e_{j k}-e_{i i} e_{i j} e_{j k}=e_{i j} e_{j k}-e_{i i}\left(e_{i k}-e_{j k} e_{i j}\right) \\
& =e_{i j} e_{j k}-e_{i i} e_{i k}+e_{i i} e_{j k} e_{i j}=e_{i j} e_{j k}-e_{i i} e_{i k} . \\
e_{i j} e_{j i} & =e_{i i} e_{i j}
\end{aligned}
$$

for $I=\sum e_{k k}$ and by (14),

$$
\begin{aligned}
e_{i j} e_{j j} & =I e_{i j} e_{j j}=\left(e_{i i}+e_{j j}\right) e_{i j} e_{j j}=e_{i i}\left(e_{i j} e_{j j}\right)+\left(e_{j j} e_{i j}\right) e_{j j} \\
& =e_{i i}\left(e_{i j}-e_{j j} e_{i j}\right)+\left(e_{i j}-e_{i j} e_{j j}\right) e_{j j} \\
& =e_{i i} e_{i j}-0+e_{i j} e_{j j}-e_{i j} e_{j j}=e_{i i} e_{i j} .
\end{aligned}
$$

Combining (16a), (16b), and (12), we get

$$
\begin{aligned}
a_{i l} & =e_{i j} e_{j l}=e_{i k} e_{k l} \quad \text { for all } j \text { and } k \text { if } i \neq l . \\
e_{i j} e_{j k} e_{k i} & =e_{i l} e_{l m} e_{m i}=e_{i m} e_{m i} e_{i i}=a_{i i} \quad(i \neq j, k, l, m),
\end{aligned}
$$

for by (16), $e_{i j} e_{j k} e_{k i}=e_{i j} e_{j m} e_{m i}=e_{i} e_{l m} e_{m i}$. In particular, this shows that the definition of $a_{i i}$ is independent of $j$.

For $k \neq i, j, l, e_{i j} a_{k l}=e_{i j} e_{k k} e_{k l}=0$.

For $k \neq i, j, e_{i j} a_{k k} \equiv e_{i j} a_{k j} e_{j k}=0 e_{j k}=0$.

For $i \neq j, k, e_{i j} a_{i k} \equiv e_{i j} e_{i j} e_{j k}=0$ by (16) and (15).

For $i \neq j, e_{i j} a_{i i} \equiv e_{i j} a_{i j} e_{j i}=0 e_{j i}=0$.

For $j \neq k, e_{i j} a_{j k} \equiv e_{i j} e_{j k} e_{k k}=a_{i k}$ by (16) or (17).

For $i \neq j$,

$$
\begin{aligned}
e_{i j} a_{j j} & \equiv e_{i j} e_{j i} e_{i i} e_{i j}=e_{i j} e_{j i} e_{i j} e_{j i} \\
& =e_{i j}\left(e_{i i}+e_{j i}-e_{i j} e_{j i}\right) e_{j j}=0+e_{i j} e_{j j}-0 \equiv a_{i j} .
\end{aligned}
$$

For $i \neq j, e_{i j} a_{i j}=e_{i i} e_{i i} e_{i j}=e_{i i} e_{i j}=a_{i j}$.

Hence, $e_{i i} a_{i i}=e_{i i} e_{i j} a_{j i}=e_{i j} e_{j j} a_{j i}=e_{i j} a_{j i}=a_{i i}$. Similar formulas hold for $a_{i j} e_{k l}$, by (16) and symmetry. Summarizing,

$$
e_{i j} a_{k l}=\delta_{j k} a_{i l}=a_{i j} e_{k l}
$$

For $j \neq i, k, a_{i j} a_{k l} \equiv e_{i j} e_{j j} a_{k l}=0$.

For $i \neq j, a_{i i} a_{j k} \equiv e_{i j} e_{j j} e_{j i} a_{j k}=e_{i j} e_{j i} e_{i i} a_{j k}=0$.

For $i \neq j, a_{i j} a_{j k} \equiv e_{i j} e_{j j} a_{j k}=e_{i j} a_{j k}=a_{i k}$.

Hence, for $i \neq j, a_{i i} a_{i j} \equiv a_{i j} e_{j i} a_{i j}=a_{i j} a_{j j}=a_{i j}$.

Likewise, $a_{i i} a_{i i} \equiv e_{i j} a_{j i} a_{i i}=e_{i j} a_{j \imath}=a_{i i}$. Summarizing,

$$
a_{i j} a_{k l}=\delta_{j k} a_{i l}
$$

(all $i, j, k, l$ ).

For $i \neq k, b_{i j} a_{k l} \equiv\left(e_{j i}-a_{j i}\right) a_{k l}=e_{j i} a_{k l}-a_{j i} a_{k l}=0$.

For all $i, j, b_{i j} a_{i k} \equiv\left(e_{j i}-a_{j i}\right) a_{i k}=a_{j k}-a_{j l}=0$. By symmetry,

$$
b_{i j} a_{k l}=0=a_{i j} b_{k l}
$$

(all $i, j, k, l$ ). 
For $i \neq j, b_{i j} \equiv e_{j i}-a_{j i}=e_{j i} I-a_{j i}=e_{j i} e_{i i}+e_{j i} e_{j j}-e_{j i} e_{i i}=e_{j i} e_{j j}$.

Hence if $j \neq i, k, l, b_{i j} b_{k l}=e_{j i} e_{j j}\left(e_{l k}-a_{l k}\right)=0$.

For $j \neq k, i, b_{i j} b_{k j}=e_{j i} e_{j j} e_{j k} e_{j j}=e_{j i} e_{j k} e_{k k} e_{j j}=0$.

For $i \neq k, b_{i j} b_{j k} \equiv\left(e_{j i}-a_{j i}\right)\left(e_{k j}-a_{k j}\right)=e_{j i} e_{k j}=e_{k i}-e_{k j} e_{j i}=b_{i k}$.

For $i \neq j$,

$$
\begin{aligned}
b_{i j} b_{j i} & =e_{j i} e_{j j} e_{i j} e_{i i}=e_{j i}\left(e_{i j}-e_{i j} e_{j i}\right) e_{i i}=e_{j i} e_{i j} e_{i i} \\
& =\left(e_{i i}+e_{i j}-e_{i j} e_{j i}\right) e_{i i}=e_{i i}+0-a_{i i} \equiv b_{i i} .
\end{aligned}
$$

For all $i$,

$$
\begin{aligned}
b_{i i} b_{i i} & =\left(e_{i i}-a_{i i}\right)\left(e_{i i}-a_{i i}\right) \\
& =e_{i i}-2 a_{i i}+a_{i i}=b_{i i} .
\end{aligned}
$$

For $k \neq i, j, b_{i j} b_{k k}=\left(e_{j i}-a_{j i}\right)\left(e_{k k}-a_{k k}\right)=0$. By symmetry, $b_{i i} b_{j k}=0$ for $i \neq j, k$. Summarizing,

$$
\left.b_{i j} b_{k l}=\delta_{j k} b_{i l} \quad \text { (all } i, j, k, l\right) .
$$

Thus by (19)-(21), the $a_{i j}$ multiply among themselves like matrix units, as do the $b_{i j}$, while $a_{i j} b_{k l}=0=b_{i j} a_{k l}$. Hence the subalgebra of $J_{u}(X)$ generated by the $a_{i j}$ and $b_{k l}$ is $M \oplus M$. But this subalgebra contains $a_{i j}+b_{j i}=e_{i j}$, for all $i$ and $j$, so the subalgebra is the whole of $J_{u}(X)$, proving the theorem. In obtaining the products we have allowed for the possibility that all the subscripts are distinct. But nowhere have we assumed the existence of subscripts which do not appear explicitly in the factors, except that if only a single subscript appeared, then we assumed the existence of a second. Hence the theorem holds for $n=2,3, \cdots$.

TheOREM 7( $\left.{ }^{9}\right)$. If $X_{S}$ is the special Jordan algebra of all symmetric $n$ by $n$ matrices $(n \geqq 2)$, then $J_{u}\left(X_{S}\right)$ is isomorphic to the $n$ by $n$ total matrix algebra over the same field.

Proof. Let $E_{i j}$ be the matrix with 1 in the $i$ th row and $j$ th column, and also in the $i$ th column and $j$ th row, and 0 elsewhere. In the notation of the proof of Theorem 6, $E_{i i} \equiv e_{i i}, E_{i j} \equiv E_{j i}=e_{i j}+e_{j i}(i \neq j)$. In $X_{S}$, the multiplication table is, by (3),

$$
\begin{array}{lr}
E_{i j} \cdot E_{k l}=0 & \text { if } i \neq k, l, \text { and } j \neq k, l ; \\
E_{i j} \cdot E_{j l}=E_{i l} / 2 & \text { if } i \neq l ; \\
E_{i j} \cdot E_{i j}=E_{i i}+E_{j i} & \text { if } i \neq j ; \\
E_{i i} \cdot E_{i i}=E_{i i} . &
\end{array}
$$

Set

$$
A_{i i} \equiv E_{i i} ; \quad A_{i j} \equiv E_{i j} E_{j j} \quad(i \neq j)
$$

(9) This example was first studied by F. D. Jacobson and N. Jacobson; cf. [13]. 
We seek the multiplication table for $J_{u}\left(X_{S}\right)$.

$$
E_{i j} E_{k \boldsymbol{k}}=0
$$

for, since $E_{k k}$ is idempotent,

$$
\begin{aligned}
E_{i j} E_{k k} & =E_{i j} E_{k k} E_{k k}=\left\{2\left(E_{i j} \cdot E_{k k}\right)-E_{k k} E_{i j}\right\} E_{k k} \\
& =\left(0-E_{k k} E_{i j}\right) E_{k k}=-E_{k k}\left(0-E_{k k} E_{i j}\right)=E_{k k} E_{k k} E_{i j}=E_{k k} E_{i j} ;
\end{aligned}
$$

but also $E_{i j} E_{k k}=0-E_{k k} E_{i j}$; adding, we get (24). Likewise,

$$
E_{k k} E_{i j}=0
$$

Hence $A_{i i} A_{j j}=\delta_{i j} A_{i i}$.

$$
E_{i j} E_{j k}=E_{i i} E_{i k}
$$

for we may suppose $i \neq j$ and then by (24) and (22),

$$
\begin{aligned}
0 & =E_{i j}\left(E_{i i} E_{j k}\right)=\left(E_{i j} E_{i i}\right) E_{j k}=\left(E_{i j}-E_{i i} E_{i j}\right) E_{j k} \\
& =E_{i j} E_{j k}-E_{i i}\left(E_{i j} E_{j k}\right)=E_{i j} E_{j k}-E_{i i}\left(E_{i k}-E_{j k} E_{i j}\right) \\
& =E_{i j} E_{j k}-E_{i i} E_{i k}+\left(E_{i i} E_{j k}\right) E_{i j}=E_{i j} E_{j k}-E_{i i} E_{i k}+0,
\end{aligned}
$$

and transposition gives (26).

$$
E_{i j} E_{i j}=E_{i i}+E_{j i}
$$

for $2 E_{i j} E_{i j}=E_{i j} E_{i j}+E_{i j} E_{i j}=2\left(E_{i j} \cdot E_{i j}\right)=2\left(E_{i i}+E_{j j}\right)$.

For $j \neq i, k, A_{i i} A_{j k} \equiv E_{i i} E_{j k} E_{k k}=E_{i i} E_{j j} E_{j k}=0$.

For $i \neq j, \quad A_{i i} A_{i j}=E_{i i} E_{i i} E_{i j}=E_{i i} E_{i j}=A_{i j}$. Similarly, for $i \neq k, A_{i j} A_{k k}$ $=\delta_{j k} A_{i k}$.

For $j \neq i, k, A_{i j} A_{k l} \equiv E_{i j} A_{j j} A_{k l}=0$.

For $i, j, k$ distinct, by (23) and (26),

$$
\begin{aligned}
A_{i j} A_{j k} & \equiv E_{i j} A_{j j} A_{j k}=E_{i j} A_{j k}=E_{i j} E_{j k} E_{k k}=E_{i k} E_{k k} E_{k k} \\
& =E_{i k} E_{k k} \equiv A_{i k} .
\end{aligned}
$$

By (27), for $i \neq j$,

$$
\begin{aligned}
A_{i j} A_{j i} & =E_{i j} E_{j j} E_{j j} E_{j i}=E_{i j} E_{j j} E_{i j}=E_{i i} E_{i j} E_{i j} \\
& =E_{i i}\left(E_{i i}+E_{j i}\right)=A_{i i} .
\end{aligned}
$$

Summarizing,

$$
A_{i j} A_{k l}=\delta_{j k} A_{i l}
$$

(all $i, j, k, l$ ).

Hence the subalgebra of $J_{u}\left(X_{S}\right)$ generated by the $A_{i j}$ is a total matrix algebra. But this subalgebra contains

$$
A_{i j}+A_{j i}=E_{i j} E_{i j}+E_{j i} E_{i i}=E_{i j}\left(E_{i i}+E_{j j}\right)=E_{i j} I=E_{i j}
$$

and is therefore the whole of $J_{u}\left(X_{S}\right)$ and the theorem holds. 
TheOREM 8. If $X_{H}$ is the special Jordan algebra of all Hermitian $n$ by $n$ matrices $(n \geqq 2)$ over the complex numbers, then $J_{u}\left(X_{H}\right)$ is isomorphic to the $n$ by $n$ total matrix algebra over the complex numbers.

Proof. Using the previous notation and $(-1)^{1 / 2}=i$, let

$$
B_{i j} \equiv-B_{j i} \equiv i\left(e_{i j}-e_{j i}\right) \quad \text { for } i \neq j .
$$

In $X_{H}$, the multiplication table is, by (3), given by (22), commutativity, and

$$
\begin{array}{lr}
E_{i j} \cdot B_{k l}=0 & \text { if } k \neq i, j, l \text { and } l \neq i, j, k ; \\
E_{i j} \cdot B_{i j}=0 & \text { where } i \neq j ; \\
E_{i j} \cdot B_{j k}=B_{i k} / 2 & \text { where } j \neq k ; \\
B_{i j} \cdot B_{k l}=0 & \text { if } i, j, k, l \text { are all distinct; } \\
B_{i j} \cdot B_{i j}=E_{i i}+E_{j j} & \text { where } i \neq j ; \\
B_{i j} \cdot B_{j k}=-E_{i k} / 2 & \text { if } i, j, k \text { are distinct. }
\end{array}
$$

In addition to (23), set (for $i \neq j$ )

$$
P_{i j} \equiv E_{i i} B_{i j}, \quad P_{i i} \equiv E_{j i} P_{j i} \equiv E_{i j} E_{j i} B_{j i}
$$

$P_{i i}$ will be shown in (34) to be independent of $j$.

$$
E_{i i} B_{j k}=0
$$

$(i, j, k$ distinct),

for by (29),

$$
\begin{aligned}
E_{i i} B_{j k} & =E_{i i} E_{i i} B_{j k}=E_{i i}\left(0-B_{j k} E_{i i}\right)=-\left(E_{i i} B_{j k}\right) E_{i i} \\
& =-\left(0-B_{j k} E_{i i}\right) E_{i i}=B_{j k} E_{i i},
\end{aligned}
$$

while $E_{i i} B_{j k}=0-B_{j k} E_{i i}$; addition yields (31). Similarly,

$$
\begin{aligned}
& B_{i j} E_{k k}=0 \\
& E_{i j} B_{j k}=E_{i i} B_{i k} \equiv P_{i k}
\end{aligned}
$$

$(k \neq i, j)$,

for we may suppose $i \neq j$, and then

$$
\begin{gathered}
E_{i j} B_{j k}=I E_{i j} B_{j k}=\left(E_{i i}+E_{i j}\right) E_{i j} B_{j k}=E_{i i} E_{i j} B_{j k}+E_{j j} E_{j i} B_{j k} \\
=E_{i i}\left(B_{i k}-B_{j k} E_{i j}\right)+E_{j i} E_{i i} B_{j k}=E_{i i} B_{i k}-0+0 \\
E_{i j} E_{j j} B_{j i}=E_{i k} E_{k k} B_{k i}
\end{gathered}
$$

(and hence $P_{i i}$ is independent of $j$ ), for

$$
\begin{aligned}
E_{i j} E_{j j} B_{j i} & =E_{i j} E_{j k} B_{k i}=E_{i k} E_{k k} B_{k i} \\
B_{i j} B_{i j} & =E_{i i}+E_{j j}
\end{aligned}
$$$$
(i \neq j)
$$

for $2 B_{i j} B_{i j}=B_{i j} B_{i j}+B_{i j} B_{i j}=2\left(B_{i j} \cdot B_{i j}\right)=2\left(E_{i i}+E_{j j}\right)$. 
For $k \neq i, j, l, E_{i j} P_{k l} \equiv E_{i j} E_{k k} B_{k l}=0$.

For $k \neq i, j, E_{i j} P_{j k} \equiv E_{i j} E_{j j} B_{j k}=E_{i i} E_{i j} B_{j k}=E_{i i} E_{i i} B_{i k}=E_{i i} B_{i k} \equiv P_{i k}$.

For $i, j, k$ distinct, $E_{i j} P_{k k} \equiv E_{i j} E_{j k} P_{j k}=E_{i i} E_{i k} P_{j k}=0$.

For $i \neq k, E_{i i} P_{k k} \equiv E_{i i} E_{i k} P_{i k}=E_{i k} E_{k k} P_{i k}=0$.

For $i \neq j, E_{i j} P_{j j} \equiv E_{i j} E_{i j} E_{i i} B_{i j}=\left(E_{i i}+E_{j j}\right) E_{i i} B_{i j}=E_{i i} B_{i j} \equiv P_{i j}$.

For all $j, E_{j j} P_{j j} \equiv E_{j j} E_{j i} E_{i i} B_{i j}=E_{j i} E_{i i} E_{i i} B_{i j}=E_{j i} E_{i i} B_{i j} \equiv P_{j j}$.

Thus $E_{i j} P_{k l}=\delta_{j k} P_{i l}+\delta_{i k} P_{j l}$ if $i \neq j$, and $A_{i i} P_{j k} \equiv E_{i i} P_{j k}=\delta_{i j} P_{i k}$.

Hence, for $i \neq j, A_{i j} P_{k l} \equiv E_{i j} E_{j j} P_{k l}=\delta_{j k} E_{i j} P_{j l}=\delta_{j k} P_{i l}$. But in spite of the unsymmetric appearance of $(30), P_{i j}$ can be written in a similar form with the $E$ 's after the $B$ 's:

$$
\begin{aligned}
P_{i j} & \equiv E_{i i} B_{i j}=B_{i j}-B_{i j} E_{i i}=B_{i j}\left(E_{i i}+E_{j j}\right)-B_{i j} E_{i i}=B_{i j} E_{j i} \\
P_{i i} & \equiv E_{i j} P_{j i}=E_{i j} B_{j i} E_{i i}=\left(0-B_{j i} E_{i j}\right) E_{i i}=B_{i j} E_{j i} E_{i i} \\
& =B_{i j} E_{j j} E_{j i}=P_{i j} E_{i j} .
\end{aligned}
$$

Summarizing previous formulas, and those symmetric to them in view of (36),

$$
A_{i j} P_{k l}=\delta_{j k} P_{i l}=P_{i j} A_{k l}
$$

(all $i, j, k, l)$.

For $j \neq i, k, P_{i j} P_{k l}=B_{i j} E_{j j} P_{k l}=B_{i j} 0=0$.

For $i, j, k$ distinct,

$$
\begin{aligned}
P_{i j} P_{j k} & =B_{i j} E_{j j} E_{j j} B_{j k}=B_{i j} E_{j j} B_{j k}=P_{i j} B_{j k} \\
& =E_{i i} B_{i j} B_{j k}=E_{i i}\left(-E_{i k}-B_{j k} B_{i j}\right)=-A_{i k} .
\end{aligned}
$$

Similarly, if $i \neq j, P_{i j} P_{j i}=E_{i i} B_{i j} B_{j i}=E_{i i}\left(-E_{i i}-E_{j j}\right)=-A_{i i}$.

For $i \neq j, P_{i i} P_{j k} \equiv E_{j i} P_{j i} P_{j k}=E_{j i} 0=0$.

For $i \neq j, P_{i i} P_{i j} \equiv E_{j i} P_{j i} P_{i j}=E_{j i}\left(-E_{j j}\right)=-E_{i j} E_{j j} \equiv-A_{i j}$. Similarly, for $i \neq j, P_{i j} P_{j j}=-A_{i j}$.

For $i \neq j, P_{i i} P_{j j}=E_{j i} P_{j i} P_{j j}=E_{j i} 0=0$.

For all $i, \quad P_{i i} P_{i i}=E_{j i} P_{j i} P_{i i}=E_{j i}\left(-A_{j i}\right)=-E_{j i} E_{j i} E_{i i}=-\left(E_{i i}+E_{j j}\right) E_{j j}$ $=-A_{j j}$. Summarizing,

$$
\left.P_{i j} P_{k l}=-\delta_{j k} A_{i l} \quad \text { (all } i, j, k, l\right) .
$$

By (28), (37), and (38), the subalgebra of $J_{u}\left(X_{H}\right)$ generated by the $A_{i j}$ and $P_{i j}$ is isomorphic to the $n$ by $n$ total matrix algebra over the complex numbers, under the correspondence $A_{i j} \leftrightarrow e_{i j}, P_{i j} \leftrightarrow i e_{i j}$. But this subalgebra contains the $E_{i j}$ as in the proof of Theorem 7, and also contains

$P_{i j}-P_{j i}=E_{i i} B_{i j}-E_{j j} B_{j i}=E_{i i} B_{i j}+E_{j j} B_{i j}=\left(E_{i i}+E_{j j}\right) B_{i j}=I B_{i j}=B_{i j}$ and hence is the whole of $J_{u}\left(X_{H}\right)$. Thus the theorem holds.

TheOREM 9. If $X_{Q}$ is the special Jordan algebra of all Hermitian $n$ by $n$ matrices $(n \geqq 3)$ over the quaternions, then $J_{u}\left(X_{Q}\right)$ is isomorphic to the $n$ by $n$ total matrix algebra over the quaternions.

Proof. Using the previous notation and denoting the quaternion units by 
$i, j, k$, set in addition

$$
C_{i j} \equiv j\left(e_{i j}-e_{j i}\right) \equiv-C_{i i} ; \quad D_{i j} \equiv k\left(e_{i j}-e_{j i}\right) \equiv-D_{j i} \quad(i \neq j) .
$$

In $X_{Q},(29)$ holds, as do similar formulas with $B$ replaced by $C$ and by $D$; also

$$
\begin{array}{lr}
B_{i j} \cdot C_{k l}=0, C_{i j} \cdot D_{k l}=0, D_{i j} \cdot B_{k l}=0 & (i, j, k, l \text { distinct }) ; \\
B_{i j} \cdot C_{j k}=D_{i k} / 2, C_{i j} \cdot D_{j k}=B_{i k} / 2, D_{i j} \cdot B_{j k}=C_{i k} / 2 & (i, j, k \text { distinct }) ; \\
B_{i j} \cdot C_{i j}=0, C_{i j} \cdot D_{i j}=0, D_{i j} \cdot B_{i j}=0 & (i \neq j) .
\end{array}
$$

In addition to (23) and (30), set (for $i \neq j$ )

$$
\begin{array}{ll}
Q_{i j} \equiv E_{i i} C_{i j}, & Q_{i i} \equiv E_{j i} Q_{j i} \equiv E_{i j} E_{j j} C_{j i}, \\
R_{i j} \equiv E_{i i} D_{i j}, & R_{i i} \equiv E_{j i} R_{j i} \equiv E_{i j} E_{j j} D_{j i} .
\end{array}
$$

Just as for (30), $Q_{i i}$ and $R_{i i}$ are independent of $j$. Likewise, formulas (31)-(38) hold with $P$ replaced by $Q$ and by $R$; it remains only to determine such products as $P_{i j} Q_{k l}$ and $Q_{i j} P_{k l}$.

$$
B_{i j} C_{j k}=R_{i k}
$$

for, by the analogues of (31)-(32),

$$
\begin{aligned}
B_{i j} C_{j k} & =B_{i j} I C_{i k}=B_{i j} E_{j j} C_{j k}=\left(-B_{j i} E_{j j}\right) C_{j k} \\
& =-\left(B_{j i}-E_{j j} B_{j i}\right) C_{i k}=B_{i j} C_{j k}-E_{j j} B_{i j} C_{j k} \\
& =\left(E_{i i}+E_{j j}\right) B_{i j} C_{j k}-E_{j j} B_{i j} C_{j k}=E_{i i} B_{i j} C_{j k} \\
& =E_{i i}\left(D_{i k}-C_{j k} B_{i j}\right)=E_{i i} D_{i k} \equiv R_{i k} . \\
B_{i j} C_{j i} & \equiv-B_{i j} C_{i j}=R_{i i}+R_{j i}
\end{aligned}
$$

since by (41) we have, for 3 by 3 matrices or larger,

$$
\begin{aligned}
B_{i j} C_{j i} & =I B_{i j} C_{j i}=\left(E_{i i}+E_{j j}\right) B_{i j} C_{j i}=E_{i i} B_{i j} C_{j i}+E_{j j} B_{j i} C_{i j} \\
& =E_{i k} B_{k j} C_{j i}+E_{j k} B_{k i} C_{i j}=E_{i k} R_{k i}+E_{j k} R_{k j}=R_{i i}+R_{j j} .
\end{aligned}
$$

For $j \neq k, P_{i j} Q_{k l}=\left(P_{i j} I\right) Q_{k l}=P_{i j} E_{j j} Q_{k l}=0$.

For $i, j, k$ distinct, $P_{i j} Q_{j k}=E_{i i} B_{i j} C_{j k} E_{k k}=E_{i i} R_{i k} E_{k k}=R_{i k} E_{k k}=R_{i k}$.

Similarly, for $j \neq i, P_{i j} Q_{j i}=E_{i i}\left(R_{i i}+R_{j j}\right) E_{i i}=R_{i i}$.

For $i \neq j, P_{i i} Q_{i j} \equiv E_{i j} P_{j i} Q_{i j}=E_{i j} R_{j j}=R_{i j}$.

For $i \neq j, P_{i j} Q_{j j} \equiv P_{i j} Q_{j i} E_{j i}=R_{i i} E_{i j}=R_{i j}$.

For all $i, P_{i i} Q_{i i} \equiv E_{i j} P_{j i} Q_{i i}=E_{i j} R_{j i}=R_{i i}$.

Thus $P_{i j} Q_{k l}=\delta_{j k} R_{i l}$. Similarly, $Q_{i j} P_{k l}=-\delta_{j k} R_{i l}$. Similar formulas hold for $Q_{i j} R_{k l}, R_{i j} Q_{k l}, R_{i j} P_{k l}, P_{i j} R_{k l}$. Thus the $A_{i j}, P_{i j}, Q_{i j}$, and $R_{i j}$ multiply in a manner isomorphic to the total matrix algebra over the quaternions, under the correspondence $A_{i j} \leftrightarrow e_{i j}, P_{i j} \leftrightarrow i e_{i j}, Q_{i j} \leftrightarrow j e_{i j}, R_{i j} \leftrightarrow k e_{i j}$. The theorem follows as before. Unlike the previous proofs, the proof of (42) assumes the existence of three different subscripts. Indeed, we show next that Theorem 9 is false for $n=2 ; J_{u}\left(X_{Q}\right)$ has order $4 n^{2}$ if $n>2$, but order $8 n^{2}$ if $n=2$. 
TheOREM 10( $\left.{ }^{10}\right)$. If $X_{2}$ is the special Jordan algebra of all Hermitian 2 by 2 matrices over the quaternions, then $J_{u}\left(X_{2}\right)$ is the direct sum $M_{Q} \oplus M_{Q}$ where $M_{Q}$ is the associative algebra of all 2 by 2 matrices over the quaternions.

Proof. Let $I=e_{11}+e_{22}, E=e_{12}+e_{21}, F=e_{11}, B=i\left(e_{12}-e_{21}\right), C=j\left(e_{12}-e_{21}\right)$, $D=k\left(e_{12}-e_{21}\right)$. Then in $J_{u}\left(X_{2}\right), I$ is a unit and, by (39),

$$
\begin{array}{rlrl}
E E & =B B=C C=D D=I, & F F=F, \\
B C & =-C B, & D B=-B D, & D C=-C D, \\
B E & =-E B, & C E=-E C, & D E=-E D, \\
B F & =B-F B, & C F=C-F C, & D F=D-F D .
\end{array}
$$

Hence every element in $J_{u}\left(X_{2}\right)$ can be written as a linear combination of products, in a specified order, of some or all or none of the symbols $E, F, B$, $C, D$ (with $I$ inserted if none of the other symbols appears in the term), no symbol appearing more than once in a term. Hence the order of $J_{u}\left(X_{2}\right)$ is at most $2^{b}=32$. We shall not assume (though it is true) that this form of an element is unique.

Set $U \equiv(I-B C D E) / 2, V \equiv(I+B C D E) / 2$. By (43), $U$ and $V$ are orthogonal idempotents which commute with $F$, and $E U=V E, E V=U E$, and similarly for $E$ replaced by $B$, by $C$, and by $D$. Set $Y \equiv V+(U-V) F, Z \equiv U$ $+(V-U) F$. Then $Y+Z=U+V=I$, so $Z=I-Y$. By calculation, $Y$ and $Z$ are orthogonal idempotents in the center of $J_{u}\left(X_{2}\right)$. Since $Y+Z=I, J_{u}\left(X_{2}\right)$ is the direct sum $Y J_{u}\left(X_{2}\right) \oplus Z J_{u}\left(X_{2}\right)$, where $Y J_{u}\left(X_{2}\right)$ contains $Y V=V-V F$, $Y V E=V E F, Y U=U F, Y U E=U F E$. These four elements generate a subalgebra $M$ of $Y J_{u}\left(X_{2}\right)$. By calculation using (43), one sees that $M$ is a 2 by 2 total matrix algebra with basis $U F, U F E, V E F, V-V F$; the unit element of $M$ is $U F+V-V F=Y$. Let $T \equiv U F E-V E F$; then by computation $Y, T B$, $T C, T D$ are a basis of a subalgebra $Q$ of $Y J_{u}\left(X_{2}\right)$ isomorphic to the quaternions, under the correspondence $Y \leftrightarrow 1, T B \leftrightarrow i, T C \leftrightarrow j, T D \leftrightarrow k$, and these elements commute with those of $M$. Hence $Y J_{u}\left(X_{2}\right)$ contains the direct product $M \otimes Q$ of order 16. Similarly $Z J_{u}\left(X_{2}\right)$ contains $M \otimes Q$. But as we noted earlier, the order of $J_{u}\left(X_{2}\right)$ is at most 32. Hence $M \otimes Q=Y J_{u}\left(X_{2}\right)$ $=Z J_{u}\left(X_{2}\right)$ and the theorem holds.

6. Jordan algebras and convex power families. We shall now show that special Jordan algebras have much the same relation to convex families of (real) matrices that Lie algebras have to Lie groups of (real) matrices. In both cases, the relation is valid only locally.

First consider the concept of a $k$-parameter family ("Schar") $\mathfrak{S}$ of matrices $X=\left(x_{i j}\right)$, in the sense of Lie. By this is meant a set of matrices, depending differentiably on $k$ "essential" parameters in a neighborhood of the identity matrix $I$. Clearly $\subseteq$ is always locally compact, in the topology defined by the norm $|X|=\left(\sum_{i, j} x_{i j}^{2}\right)^{1 / 2}$. Furthermore, if $X=I+A$ with

(10) This form of the theorem and proof was suggested by the referee. 
$|A|<1$, we may $\left.{ }^{11}\right)$ define $\ln X=A-A^{2} / 2+A^{3} / 3-\cdots$; the series is absolutely convergent. We may further define

$$
X^{t}=\exp (t \ln X)=I+Y+Y^{2} / 2 !+Y^{3} / 3 !+\cdots,
$$

where $Y=t \ln X$. The $X^{t}$ form a one-parameter Lie group, with $X^{1}=X$.

Now it is well known that if $\subseteq$ is a local Lie group, then for some $\delta>0$, $X \in S$ and $|\ln X|<\delta$ and $|t \ln X|<\delta$ imply $X^{t} \in \widetilde{S}$; this is a corollary of the existence of canonical parameters. Hence the concept of a local Lie group is a special case of the following concept of a "local power family"; and the usual concepts of infinitesimal generator and equality may be generalized to local power families as follows.

Definition. A local power family is a set $\mathfrak{S}$ of matrices which is locally compact, and such that for some $\delta_{1}>0, X \in \mathfrak{S}$ and $|\ln X| \leqq \delta_{1}$ and $|t \ln X| \leqq \delta_{1}$ together imply $X^{t} \in \mathfrak{S}$. The infinitesimal matrices of $\mathfrak{S}$ are the set $J(\widetilde{S})$ of matrices $Y$ such that $\exp (t Y) \in \mathbb{S}$ for all sufficiently small $t$. Two local power families are locally equal if and only if they coincide in some neighborhood $U_{\delta}:|X-I|<\delta$ of $I$.

LEMma 6.1. If $\mathfrak{S}$ is a local power family, and $X \in \mathfrak{S}$ and $|\ln X|<\delta_{1}$, then $\ln X \in J(\mathfrak{S})$.

Proof. $X^{t}=\exp \left(\ln X^{t}\right)=\exp (t \ln X)$. By definition of a local power family, $X^{t} \in \mathfrak{S}$ for $t$ sufficiently small. Hence by definition of $J(\mathfrak{S}), \ln X \in J(\Im)$.

We note also that from the definitions, $J(\mathfrak{S})$ is closed under scalar multiplication. Now, given $\delta$ such that $\delta<1$ and $\delta<\delta_{1}$, set $J_{\delta}(\Im) \equiv J(\Im) \cap \bar{U}_{\delta}$, where $\bar{U}_{\delta}$ is the set of $X$ with $|X-I| \leqq \delta$. But from the closure of $J(\Im)$ under scalar multiplication, $J_{\delta}(\mathfrak{S})$ is closed under scalar multiplication out to radius $\delta$. Further, since $\ln X$ and exp $Y$ are continuous and mutually inverse, and $\mathbb{S}$ is by hypothesis locally compact, $J_{\delta}(\mathfrak{S})$ is locally compact. But in view of the closure of $J(\Im)$ under scalar multiplication, $Y \in J(\Im)$ is equivalent to $t Y \in J_{\delta}(\mathfrak{S})$ for sufficiently small $t$; hence $J(\mathfrak{S})$ consists of the scalar multiples of the elements of $J_{\delta}(\Im)$, and bounded subsets of $J(\Im)$ are compact; in particular, $J(\Im)$ is closed. It is further easily shown that two local power families are locally equal if and only if they have the same set $\Im$ of infinitesimal matrices-which implies that some neighborhood of each consists of the $\exp Y[Y \in \Im,|Y|<\delta]$.

TheOREM 11. Let $\mathfrak{S}$ be any local power family of matrices. Then the infinitesimal matrices of $\mathfrak{S}$ form a Jordan algebra if and only if $\mathfrak{S}$ is "locally convex," in the sense that for some $\delta>0$, the set $\mathfrak{S}_{\delta} \equiv \mathfrak{S} \cap U_{\delta}$ is convex. (Here $U_{\delta}$ denotes the convex "sphere" of all $X$ with $|X-I|<\delta$.)

Proof. Suppose $J(S)$ is a Jordan algebra. Take $\delta<\delta_{1}$. If $X \in S_{\delta}$ then $|X-I|<\delta$ and $Y=\ln X \in J(\Im)$ and $X=\exp Y=I+Y+Y^{2} / 2+\cdots$. Since $J(\Im)$ is a Jordan algebra, it is closed under sums and powers, while we have

(11) See von Neumann [20]. 
already seen that it is closed under limits. Hence $Y+Y^{2} / 2 !+\cdots \in J(\Im)$. Thus $X=I+A$ with $A \in J(\Im)$ and $|A|<\delta$. Conversely, if $A \in J(\subseteq)$ and $|A|<\delta<1$, then $J(\Im)$ contains $Y=\ln (I+A)=A-A^{2} / 2+A^{3} / 3+\cdots$, being closed under sums, powers, and limits. If also $\delta$ is sufficiently small, then $|Y|<\delta_{1}$. By definition of $J(\Im), Z=\exp (u Y) \in \subseteq$ for sufficiently small $u$. Taking $|u|<1$ and $t=1 / u,|\ln Z|=|u Y|<\delta_{1}$ and $|t \ln Z|=|Y|<\delta_{1}$, so by definition of a local power family, $\subseteq$ contains $Z^{t}=(\exp Y)^{u t}=I+A$. We conclude that if $J(\mathfrak{S})$ is a Jordan algebra then locally $\mathfrak{S}$ consists of $U_{\delta} \cap[I+J(\Im)]$, where $I+J(\Im)$ denotes the set of all $I+A$ for $A \in J(\Im)$. But $U_{\delta}$ is convex by construction, while $I+J(\mathfrak{S})$ is convex since $J(\mathfrak{S})$ is by hypothesis an algebra. Hence $\mathfrak{S}$ is locally convex.

Conversely, suppose that $\subseteq$ is convex; we wish to show that $J(\subseteq)$ is a Jordan algebra. Since $J(\Im)$ is always closed under multiplication by scalars, we must show that $A \in J(\Im)$ and $B \in J(\Im)$ imply $A+B \in J(\Im)$ and $(A B+B A) / 2 \in J(S)$; this we now do.

If $A \in J(\mathfrak{S})$ then by definition $\exp (A t) \in \mathfrak{S}$ for sufficiently small $t$. Since $\Im$ is convex, it contains

$$
C \equiv[\exp (A t)+\exp (B t)] / 2=I+(A+B) t / 2+\left(A^{2}+B^{2}\right) t^{2} / 4+\cdots .
$$

Then

$$
\begin{aligned}
\ln C= & {\left[(A+B) t / 2+\left(A^{2}+B^{2}\right) t^{2} / 4+\cdots\right] } \\
& -\left[(A+B)^{2} t^{2} / 4+\cdots\right] / 2+\cdots ; \\
D \equiv & \ln C^{2 u / t}=(2 u / t) \ln C \\
= & u\left\{(A+B)+\left[\left(A^{2}+B^{2}\right) / 2-(A+B)^{2} / 4\right] t+\cdots\right\} .
\end{aligned}
$$

Obviously, for $t$ and $u$ sufficiently small (and $u$ independent of $t$ ), $|\ln C|<\delta_{1}$ and $|(2 u / t) \ln C|=|D|<\delta_{1}$. By definition of a local power family, $C^{2 u / t} \in S_{\text {. }}$. Then $\exp (E u) \in \subseteq$ for sufficiently small $u$, where

$$
E \equiv \ln C^{2 / t}=(A+B)+\left[\left(A^{2}+B^{2}\right) / 2-(A+B)^{2} / 4\right] t+\cdots \cdot
$$

Hence, by definition, $E \in J(S)$. Hence, since $J(\mathfrak{S})$ is closed, it contains $\lim _{t \rightarrow 0} E=A+B$. Thus $J(\Im)$ is closed under scalar multiplication and addition, so, by (44), it contains

$$
\begin{aligned}
4[E-(A+B)] / t & =4\left[\left(A^{2}+B^{2}\right) / 2-(A+B)^{2} / 4\right]+\cdots \\
& =\left[A^{2}+B^{2}-A B-B A\right]+\cdots
\end{aligned}
$$

for all sufficiently small $t$, where the dots indicate terms containing $t$ as a factor. Since $J(\mathfrak{S})$ is closed, it must therefore contain

$$
H \equiv \lim _{t \rightarrow 0} 4[E-(A+B)] / t=A^{2}+B^{2}-A B-B A .
$$

In particular, let $B=0$, noting that $0 \in J(\Im)$ by closure under scalar multi- 
plication. This gives $A^{2} \in J(\mathfrak{S})$; likewise $B^{2} \in J(\Im)$. Hence $A^{2}+B^{2}-H$

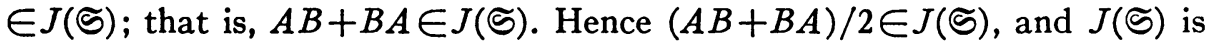
a Jordan algebra, proving the theorem.

The first half of the proof essentially applies if $\Im$ is any Jordan algebra of matrices which is closed under limits, even if $\subseteq$ is not given. For suppose $\Im$ is such an algebra. Denote by $S(\Im)$ the set of all $I+A[A \in \Im]$. We call $S(\Im)$ a power family. Suppose $X \in S(\Im)$ and $|X-I|<1$. By definition of $S(\Im), X=I+A$ for some $A \in \Im$. Then

$$
(I+A)^{t}=I+t A+t(t-1) A^{2} / 2 !+\cdots .
$$

Then $B \equiv t A+t(t-1) A^{2} / 2 !+\cdots \in \Im$ by closure, so $(I+A)^{t}=I+B \in S(\Im)$. Obviously, $S(\Im)$ is convex.

This may be regarded as the convex power family generated by $\Im$, and is the analog of the concept of a global Lie group. We thus establish a one-to-one correspondence between Jordan algebras of matrices and global convex power families.

The above discussion applies to matrices with complex coefficients, without essential change.

7. Postulates for binary mean. In connection with Jordan multiplication (3), where we take the mean of $X Y$ and $Y X$, it seems of interest to consider certain properties of means $\left({ }^{12}\right)$.

We postulate a binary operation $m$ on abstract elements $A, B, \cdots$, with the properties

$$
\begin{aligned}
A m A & =A ; \\
A m B & =B m A ; \\
(A m B) m(C m D) & =(A m C) m(B m D) .
\end{aligned}
$$

From (46) and (47) it follows immediately that

$$
(A m B) m(C m D)=(A m D) m(C m B) \text {. }
$$

Let $\mathfrak{S}$ be a system each of whose elements is an unordered set of $2^{n}$ letters (not necessarily distinct), where $n$ is a non-negative integer depending on the element. If $A \in \mathcal{S}$, denote by $l(A)$ the value which $n$ has for the particular element $A$. Define equality in $\mathfrak{S}$ thus: if $A$ and $B$ are in $\mathfrak{S}$, with $l(A)=k$ and $l(B)=n$, then $A=B$ if and only if either $k \leqq n$ and each distinct letter appears in the set $B$ exactly $2^{n-k}$ times as often as in $A$, or $n \leqq k$ and each distinct letter appears in $A$ exactly $2^{k-n}$ times as of ten as in $B . A m B$ is defined to be the unordered set consisting of each letter of $A$ repeated $2^{n}$ times and each letter of $B$ repeated $2^{k}$ times.

THEOREM 12. The system $\mathfrak{S}$ just defined is the free $\left({ }^{13}\right)$ algebra on a countable number of elements satisfying (45)-(47).

(12) For a study of means of ordinary numbers, see Huntington [12].

(13) For the general concept of free algebras, see for instance Birkhoff [8]. 
Proof. First we verify that $\subseteq$ satisfies (45)-(47). $A m A$ consists, by the above definition, of each letter of $A 2^{k}$ times, and then again each letter of $A 2^{k}$ times-in other words, of each letter of $A 2^{k+1}$ times. Then by definition of equality, $A m A=A$.

It is evident from the definitions that $A m B=B m A$.

As for (47), we observe that since the elements in $\subseteq$ are unordered sets, it is only a question of the multiplicities of the letters. Let $l(A)=k, l(B)=n$, $l(C)=p, l(D)=q$. If the set $A$ consists of the distinct letters $a_{i}$ with multiplicities $\alpha_{i}$, we may write $A=\left\{\alpha_{i} a_{i}\right\}, B=\left\{\beta_{i} b_{i}\right\}, C=\left\{\gamma_{i} c_{i}\right\}, D=\left\{\delta_{i} d_{i}\right\}$. Then by construction of $\mathfrak{S}$,

$$
\begin{array}{ll}
A m B=\left\{2^{n} \alpha_{i} a_{i}, 2^{k} \beta_{i} b_{i}\right\}, & l(A m B)=2^{k+n+1} ; \\
C m D=\left\{2^{q} \gamma_{i} c_{i}, 2^{p} \delta_{i} d_{i}\right\}, & l(C m D)=2^{p+q+1} .
\end{array}
$$

Hence

$$
(A m B) m(C m D)=\left\{^{2 n+p+q+1} \alpha_{i} a_{i}, 2^{k+p+q+1} \beta_{i} b_{i}, 2^{k+n+q+1} \gamma_{i} c_{i}, 2^{k+n+p+1} \delta_{i} d_{i}\right\},
$$

a result whose symmetry shows that it is independent of the order in which $A, B, C, D$ are taken, so that (47) holds. Thus $\subseteq$ satisfies (45)-(47).

Secondly we wish to show that any finite or countable system $\Re$ satisfying (45)-(47) is a homomorphic image of $\mathfrak{S}$. By (45), in $\Re$ we have $A m A=A$; thus by inserting repetitions of the given elements we can write any given element in $\Re$ as a mean of $2^{n}$ elements. Likewise any mean $A$ of $2^{k}$ elements of $\Re$ can be written as a mean of $2^{n}$ elements, if $k \leqq n$, simply by increasing the multiplicity of each element in $A$ by the factor $2^{n-k}$, by (45).

We desire $\Re$ to be a homomorphic image of $\mathfrak{S}$; since the letters in the sets in $\mathfrak{S}$ are unordered, we shall need that repeated means in $\Re$ are independent of the order in which the elements are taken. For a mean of two elements of $\Re$, this condition is precisely (46). For a mean of four or more elements, (47) and (48) give the result in special cases, but we must prove it in general. We proceed by induction.

Suppose it is true for $n<k(k \geqq 2)$ that the mean of $2^{n}$ elements of $\Re$ is independent of their order, and that there is given a mean $M$ of $2^{k}$ elements. Let $Q_{1}$ stand for the first one-fourth of the places in the given repeated mean, $Q_{2}$ for the second fourth of the places therein, and so on, while the first half of the places is called $H_{1}$ and the second half $H_{2}$. Then the mean of the elements of $Q_{1}$ is some element of $\Re$, the mean of those of $Q_{2}$ is another, and so on, so that we have for the given mean

$$
M=\left(Q_{1} m Q_{2}\right) m\left(Q_{8} m Q_{4}\right) .
$$

Suppose it is desired to arrange the elements involved in the mean in the order $a, b, c, \cdots$. If $a \in H_{1}$, then by induction we may move $a$ to the desired position in $H_{1}$, and so in $M$, without altering the mean. If $a \in H_{2}$, then by (46), we may interchange the elements of $H_{1}$ with the elements of $H_{2}$, so 
now $a \in H_{1}$ and as before $a$ can be put in the desired place. It is desired that $b$ follow $a$. If $b \in H_{1}$, the previous change having already been made, this can be achieved by induction. If $b \in H_{2}$, then by (46) applied to $Q_{3} m Q_{4}$, we can put $b$ in $Q_{3}$; then by (47) we can move it to $Q_{2}$, so $b \in H_{1}$ and can be handled by induction as before.

If there are only four elements in $M$, then $a$ and $b$ complete the desired order for $H_{1}$; the other elements must be in $H_{2}$ and by induction $H_{2}$ can be rearranged as desired.

If there are more than four elements in $M$, then $a$ and $b$ are both in $Q_{1}$ and $c$ is desired as the next letter. If $c \in H_{1}$, rearrange $H_{1}$ by induction. Otherwise, $c \in H_{2}$, and we can move it to $H_{1}$ (and if $Q_{1}$ is not filled, to $Q_{1}$ ) just as was done with $b$ above. By continuing in a similar manner, we can fill $Q_{1}$ with the letters which it is desired should be there, and by induction arrange them in the desired order. Then each other letter is in $Q_{2}$ or in $H_{2}$. Say $g$ is desired as the first letter of $Q_{2}$. If $g$ is in $Q_{2}$, we can move it to $Q_{3}$ by (47) without affecting $Q_{1}$ which is already arranged. If $g$ is in $Q_{4}$ we can move it to $Q_{3}$ by induction which permits rearrangement of $H_{2}$, likewise without affecting $Q_{1}$. Hence in any case we can put $g$ in $Q_{3}$. Suppose it is desired that $h$ follow $g$. If $h$ is in $Q_{2}$, we can move it to $Q_{4}$ by (48), without disturbing either $Q_{1}$ which is arranged, or $Q_{3}$, which contains $g$. By induction, we can rearrange $H_{2}$ so as to get $g$ and $h$ in $Q_{3}$. By repeating this process we can fill $Q_{3}$ with the elements which it is desired shall ultimately be in $Q_{2}$. Then by (47) we can put these elements in $Q_{2}$, and by induction we can rearrange $Q_{2}$ in the desired order. Thus $H_{1}$ is put in order; all remaining elements must be in $H_{2}$ which can then be arranged by induction.

Thus we have shown that any mean in $\Re$ can be represented as a mean of $2^{k}$ elements for some $k$, and that these elements can be rearranged in any desired order - that is, that they are essentially unordered. If $\Re$ is given, let the elements of $\Re$ constitute the letters of $\mathfrak{S}$ and let each set $A \in \mathfrak{S}$ correspond to the (essentially unordered) mean of these elements in $\Re$. If $A=B$ in $\mathfrak{S}$, then the corresponding means $A^{\prime}$ and $B^{\prime}$ in $\Re$ will be equal, for if $A=B$ in $\mathfrak{S}$ then by definition of equality in $\subseteq$ each letter in the shorter (say in $A$ ) appears with a uniformly greater multiplicity in the other; in $\Re$, by $(45)$, these two means are equal so $A^{\prime}=B^{\prime}$. Likewise, if $A$ corresponds to $A^{\prime}$ and $B$ corresponds to $B^{\prime}$ then by the construction of the correspondence $A m B$ corresponds to $A^{\prime} m B^{\prime}$. Thus $\Re$ is a homomorphic image of $\mathfrak{S}$ as desired and the theorem is proved.

But now it is clear that if $A_{1}, \cdots, A_{n}$ are any $n$ linearly independent matrices (or other vectors), and if $A m B$ is interpreted as $(A+B) / 2$, then distinct elements of $\mathfrak{S}$ (as defined above) represent different matrices. Hence (45)-(47) are a complete set of postulates for binary means.

It would be interesting to find similar postulates for ternary and $n$-ary means, but these are less relevant to Jordan algebras. 


\section{REFERENCES}

1. I. Ado, Bull. Soc. Phys.-Math. Kazan vol. 7 (1935) pp. 3-43.

2. A. A. Albert, On a certain algebra of quantum mechanics, Ann. of Math. vol. 35 (1934) pp. $65-73$.

3. - On Jordan algebras of linear transformations, Trans. Amer. Math. Soc. vol. 59 (1946) pp. 524-555.

4. - - A structure theory for Jordan algebras, Ann. of Math. vol. 48 (1947) pp. 546-567.

5. Garrett Birkhoff, On the structure of abstract algebras, Proc. Cambridge Philos. Soc. vol. 31 (1935) pp. 433-454.

6. - Representability of Lie algebras and Lie groups by matrices, Ann. of Math. vol. 38 (1937) pp. 526-532.

7. - Analytical groups, Trans. Amer. Math. Soc. vol. 43 (1938) pp. 61-101.

8. - Universal algebra, Proceedings of the Canadian Mathematical Congress, Montreal, 1946, pp. 310-326.

9. J. E. Campbell, Introductory treatise on Lie's theory of finite continuous transformation groups, Oxford, 1903.

10. E. Cartan, Les représentations linéaires des groupes de Lie, J. Math. Pures Appl. vol. 17 (1938) pp. 1-12.

11. C. Chevalley, Theory of Lie groups, Princeton, 1946.

12. E. V. Huntington, Sets of independent postulates for the arithmetic mean, the geometric mean, the harmonic mean, and the root-mean-square, Trans. Amer. Math. Soc. vol. 29 (1927) pp. $1-22$.

13. F. D. Jacobson and N. Jacobson, Classification and representation of semi-simple Jordan algebras, to be published in Trans. Amer. Math. Soc.

14. N. Jacobson, Non-commutative polynomials and cyclic algebras, Ann. of Math. vol. 35 (1934) pp. 197-208.

15. P. Jordan, J. v. Neumann, and E. Wigner, On an algebraic generalization of the quantum mechanical formalism, Ann. of Math. vol. 35 (1934) pp. 29-64.

16. G. K. Kalisch, On special Jordan algebras, Trans. Amer. Math. Soc. vol. 61 (1947) pp. 482-494.

17. G. Kowalewski, Einfilhrung in die Theorie der kontinuierlichen Gruppen, Leipzig, 1931.

18. J. C. C. McKinsey and A. Tarski, The algebra of topology, Ann. of Math. vol. 45 (1944) pp. 141-191.

19. - On closed elements in closure algebras, Ann. of Math. vol. 47 (1946) pp. 122-162.

20. J. v. Neumann, Über die analytischen Eigenschaften von Gruppen linearer Transformationen und ihrer Darstellungen, Math. Zeit. vol. 30 (1929) pp. 3-42.

21. E. Noether, Hyperkomplexe Grossen und Darstellungstheorie, Math. Zeit. vol. 30 (1929) pp. 641-692. 165.

22. A. Tarski, $A$ remark on functionally free algebras, Ann. of Math. vol. 47 (1946) pp. 163-

23. B. L. van der Waerden, Moderne Algebra, 2d. ed., vol. 2, Berlin, 1940.

24. E. Witt, Treue Darstellung Liescher Ringe, J. Reine Angew. Math. vol. 177 (1937) pp. 152-160.

HARVARD UNIVERSITY,

CAmbridge, Mass.

Tufts College,

MEdford, Mass. 